Revue d'histoire de l'Amérique française

REVUE D.HISTOIRE DE L'AMÉRIQUE FRANÇAISE

\title{
Les Canadiens et la France révolutionnaire
}

\section{Michel Brunet}

Volume 13, numéro 4, mars 1960

URI : https://id.erudit.org/iderudit/302002ar

DOI : https://doi.org/10.7202/302002ar

Aller au sommaire du numéro

Éditeur(s)

Institut d'histoire de l'Amérique française

ISSN

0035-2357 (imprimé)

1492-1383 (numérique)

Découvrir la revue

Citer cet article

Brunet, M. (1960). Les Canadiens et la France révolutionnaire. Revue d'histoire de l'Amérique française, 13(4), 467-475. https://doi.org/10.7202/302002ar d'utilisation que vous pouvez consulter en ligne.

https://apropos.erudit.org/fr/usagers/politique-dutilisation/ 


\section{LES CANADIENS ET LA FRANCE RÉVOLUTIONNAIRE}

La Révolution française et les succès militaires de la République impressionnèrent fortement les Canadiens. Ceux-ci suivirent avec intérêt la marche des événements et la France ellemême, par sa propagande, se chargea de les renseigner. ${ }^{1}$

Les classes dirigeantes du Canada français - administrateurs ecclésiastiques, seigneurs et bourgeois - s'indignèrent devant les excès de la Révolution. Spontanément portés à défendre l'ordre établi, ils se demandèrent quel vent de folie soufflait sur l'ancienne mère-patrie. Mgr Hubert constate avec effroi que la France, abandonnant ses anciens principes, s'identifie maintenant avec « un esprit d'irréligion, d'indépendance, d'anarchie, de parricide». En plus de condamner à l'exil la «saine partie des Français », n'a-t-elle pas « conduit à l'échafaud [son] vertueux souverain »? Les mauvais exemples qu'elle donne ont «justement excité l'indignation de toutes les puissances de l'Europe ». L'évêque de Québec se dit même convaincu que «le plus grand malheur qui pût arriver au Canada serait de tomber en la possession de ces révolutionnaires ». Il invite donc le clergé de la colonie à joindre ses prières aux siennes « afin d'obtenir du ciel qu'il détourne de dessus ce diocèse les malheurs innombrables dans lesquels pourraient le précipiter les nouveaux systèmes d'impiété, d'indépendance et de libertinage, s'ils venaient à y prendre racine $\otimes^{2}$

Cette prise de position traduit assez bien l'opinion générale des porte-parole officiels de la collectivité. La Révolution leur apparut, après la chute de la monarchie, comme une aventure

1 Voir Michel Brunet, \& La Révolution française sur les bords du SaintLaurent \$, Revus d'histoire de l'Amérique française, II (septembre 1957): 155-162.

2 * Circulaire à MM. les Curés à l'occasion des rumeurs de guerre $»$, 9 novembre 1793, Mandements des évêques de Québec, 2: 471-473. 
criminelle. De plus, lorsque la Grande-Bretagne fut entraînée dans la guerre contre la France (février 1793), les dirigeants canadiens se seraient rendus coupables de trahison s'ils avaient manifesté ouvertement quelque sympathie pour la République française. Combien, toutefois, durent se réjouir secrètement des défaites infligées par la France à ses ennemis, en particulier aux Anglais ? L'historien ne le saura jamais mais il a le droit de supposer qu'ils furent nombreux, même chez les classes dirigeantes.

Dans la masse du peuple, la situation fut bien différente. Les petites gens se sentirent beaucoup plus libres d'exprimer leurs véritables sentiments. Par exemple, les braves censitaires de la seigneurie de Port-Joli refusaient de prêter foi à leur seigneur lorsque celui-ci, après avoir lu ses journaux, tentait de les mettre au courant des événements qui se déroulaient en France. Selon eux c'était la propagande anglaise qui inventait ces horreurs, surtout lorsqu'on annonça quel avait été le sort de la famille royale, afin de discréditer l'ancienne mère-patrie, « un conte inventé par l'Anglais », disaient-ils. ${ }^{3}$ Cette attitude révèle à la fois la méfiance, la haine même, qu'inspiraient les Anglais et l'affection que le peuple éprouvait pour la France. Dans sa circulaire du 9 novembre 1793, l'évêque déclara qu'il ne mettait pas en doute la loyauté du clergé envers le gouvernement britannique mais qu'il craignait les réactions des « habitants de ce pays, surtout dans les campagnes, frappés du nom de Français ».

L'amour de la France et la haine de l'occupant firent oublier aux Canadiens des classes populaires les aspects discutables et les abus de la Révolution française. Leur imagination se laissa emporter par les nouvelles que les Français avaient non seulement repoussé leurs envahisseurs mais que leurs armées et leur marine avaient remporté de nombreuses victoires. Tous les documents de l'époque indiquent que les Canadiens cherchaient à connaître ce qui se passait alors en Europe et qu'ils étaient suffisamment bien renseignés. Les succès étonnants de la France

3 Fait rapporté dans Philippe Aubert de Gaspé, Mémoires (Montréal, 1930), 109. 
révolutionnaire ne pouvaient que soulever leur enthousiasme. Pourquoi auraient-ils été tristes puisque la mère-patrie était victorieuse ? Quelques-uns crurent même qu'elle viendrait les libérer. Lors de la Révolution américaine, les Canadiens avaient eu cet espoir. Il avait été déçu. Ce que la monarchie déclinante n'avait pas osé entreprendre pourquoi la République triomphante ne le réaliserait-elle pas ? La propagande des représentants officiels de la France aux Etats-Unis, aidés par quelques Américains et Canadiens qui avaient embrassé la cause des républicains français, encouragea ces espérances.

Le peuple se montra particulièrement frondeur et s'agita au moindre prétexte. Un paroissien de Saint-François-de-la-Rivièredu-Sud refuse encore, trente ans après le traité de Paris, de reconnaître le roi d'Angleterre pour son légitime souverain. L'évêque ordonne au curé de ne pas le marier. Afin de pouvoir épouser celle qu'il aime, il décide de se déclarer «fidèle royaliste ». Mais quelques jours plus tard, il semble vouloir revenir à son ancienne opposition. ${ }^{4}$ Le curé de Saint-Thomas-de-Montmagny ne doute pas que la population est «contre le gouvernement » et prête à se révolter. ${ }^{5}$ Le gouverneur se rend compte qu'il sera très difficile de mettre en vigueur une loi de milice. ${ }^{6}$ Il voyait juste car lorsque les autorités militaires de Québec tentèrent, au mois de mai 1794, de tirer au sort les noms des miliciens destinés à former une force de deux mille hommes qui auraient été à la disposition du gouvernement en cas d'urgence, les miliciens convoqués à cette fin se mutinèrent. L'un des meneurs, au moment où l'on commença à procéder au tirage au sort, lança son chapeau dans les airs en criant: "Vivent les Français ! ${ }^{7}$ Sur quarantedeux paroisses du district de Québec, comptant un minimum de 7,000 hommes aptes à servir dans la milice, seulement huit paroisses, groupant environ neuf cents miliciens, répondirent à l'appel

${ }^{4}$ Lettres de Mgr Hubert au curé P.-L. Bédard, les 13, 14 et 19 août 1793, Rapport de l'archiviste de la province de Québec pour les années 1930-1931 (RAPQ), 289.

5 Voir Léon Trépanier, «Dambourgès le Balafré», Les Cahiers des Dix (Montréal, 1954), 241.

6 Dorchester à Dundas, 18 novembre 1793, AC, Q 67: 7 .

7 Rapport de Dorchester à Dundas, 25 mai 1794, AC, Q 71-1: 5-7. 
du gouvernement. ${ }^{8}$ Les habitants des districts des Trois-Rivières et de Montréal réagirent de la même façon. A Montréal, le 25 avril 1794, une véritable émeute éclata lorsque le shérif tenta d'exposer un condamné au pilori. Il se vit forcé de le libérer. ${ }^{9}$

Les agitateurs et les émeutiers tiennent un langage très violent qu'ils empruntent aux révolutionnaires de Paris. On menace, par exemple, d'incendier les maisons des paysans qui refusent de se joindre aux opposants et de mettre «leurs têtes au bout des bâtons $\gg^{10}$ Un propagandiste, dénonçant le loyalisme de l'évêque et des prêtres, s'écrie: « Jusqu'au clergé qui s'en mêle, une bande d'ivrognes qui ne tarderont pas à être servis, comme le clergé a été servi en France » $^{11}$ Le procureur général Monk est scandalisé de constater que les Canadiens aient réussi à s'approprier en si peu de temps les expressions et le vocabulaire des sans-culottes et des Jacobins. ${ }^{12}$ Dorchester en conclut qu'ils ont les yeux tournés vers Paris et qu'ils attendent une intervention française. ${ }^{13}$ Cependant, le gouvernement, se rendant compte que la masse du peuple lui est hostile, n'ose se montrer trop sévère de peur de provoquer un soulèvement général. ${ }^{14}$ Le gouverneur rappelle au ministre que les Canadiens se sentent forts car ils se savent les plus nombreux. ${ }^{15}$ Le procureur général conseille au gouvernement impérial d'envoyer des soldats ${ }^{16}$ et favorise, à travers toute la province, l'organisation d'une Association loyale dont se voient obligés de faire partie les représentants les plus influents de la population. C'est un moyen habile de les compromettre, aux yeux de celle-ci, en faveur du gouvernement et de priver la masse de ses chefs naturels. ${ }^{17}$

8 Rapport de Monk à Dorchester, 29 mai 1794, AC, Q 69-1: 4.

9 Rapport du shérif Gray, 9 juin 1794, AC, Q 69-2: 309-312; Monk à Dorchester, 18 juin 1794, AC, Q 69-1: 54.

10 Déposition de Louis Savard, habitant de Charlesbourg, 29 mai 1794, AC, Q 69-1: 17.

11 Déposition de William Boutillier, 24 mai 1794, AC, Q 71-1: 15.

12 Monk à Dundas, 30 mai 1794, AC, Q 69-2: 256.

13 Dorchester à id., 5 juin 1794, AC, Q 69-1: 1-2.

14 Id. à $i d$., 25 mai 1794, AC, Q 71-1: 6; Monk à Dorchester, 29 mai 1794, AC, Q 69-1: 8.

15 Dorchester à Dundas, 25 mai 1794, AC, Q 71-1: 7.

16 Monk à id., 30 mai 1794, AC, Q 69-2: 258.

17 Id. à id., 5 juillet 1794, AC, Q 69-2: 324-325; \& L'Association loyale de Montréal (1794) », RAPQ (1948-1949), 253-273. 
A l'automne de 1796, une nouvelle fièvre d'agitation s'empara de la population. Lors de l'incendie de l'église et du couvent des Récollets de la ville de Québec, la rumeur se répandit que les autorités anglaises étaient responsables de ce désastre. ${ }^{18} \mathrm{Au}$ cours de l'été, le seigneur de Port-Joli rapporta que deux de ses tenanciers l'avaient «insulté grièvement » et lui avaient tenu un langage révolutionnaire, criant «vive la liberté » et se réclamant de la « convention nationale ${ }^{19}$ La mise en vigueur d'une loi récente sur les chemins provoqua une campagne systématique contre le gouvernement et ses représentants. Des messagers parcoururent les campagnes demandant aux paysans de s'opposer à la nouvelle loi et de tenir des assemblées à cette fin. Une fois de plus, on menaça d'incendier les maisons et les granges de ceux qui refuseraient de prendre part à ces manifestations de protestation.

Le 4 octobre, une foule en colère, réunie à la Place d'Armes de Montréal, arracha des mains du shérif un agitateur qu'il venait d'arrêter et tentait de conduire chez un juge de paix. Dans quelques paroisses, les assemblées convoquées pour nommer les inspecteurs des chemins dégénérèrent en bagarres. Les magistrats venus pour disperser les gens furent insultés et parfois attaqués. ${ }^{20}$ Les juges de paix de Montréal préviennent le gouverneur que les autorités civiles de la ville et de la région sont incapables de contraindre la population à l'obéissance si celle-ci persiste dans les mêmes dispositions. ${ }^{21}$ En réponse à cette démarche, le gouvernement se décide à mettre la troupe à la disposition des magistrats en les priant de n'y recourir qu'après avoir épuisé les autres moyens de persuasion. ${ }^{22}$ Le 24 octobre, la crise atteignit son paroxysme dans la région de Montréal. Les officiers civils ne cherchent même plus à se faire obéir. L'arrivée de deux régi-

18 Gaspé, Mémoires, 71.

19 Appel de Philippe-Ignace Aubert de Gaspé aux juges de paix de la région, 7 juillet 1796, Bulletin des recherches historiques, 42 (1936): 379. 20 Rapport du procureur général Sewell, remis le 12 mai 1797, Rapport des Archives publiques du Canada (RAC), (Ottawa, 1891), note D, 73-78. 10-11.

21 Juges de paix de Montréal à Prescott, 13 octobre 1796, AC, Q 78: 12-13.

22 Ryland aux juges de paix de Montréal, 15 octobre 1796, AC, Q 78: 
ments, dépêchés par le gouverneur, rétablit finalement la situation. ${ }^{23}$ A Québec, il y eut aussi des manifestations hostiles au gouvernement mais le mouvement d'opposition fut beaucoup moins violent. Le gouverneur attribua ce calme relatif au fait que les magistrats québécois s'étaient montrés plus fermes que ceux de Montréal. ${ }^{24}$ Il ne faut pas oublier que la présence du gouverneur et de la garnison facilitait leur tâche !

La France révolutionnaire n'était pas absente de la pensée des agitateurs et des émeutiers. Prescott informa le ministre que «tout récemment, la nouvelle de la présence d'une escadre française sur les côtes a été accueillie avec une satisfaction marquée par beaucoup de gens d'ici, et qu'elle a encouragé les désordres à Montréal ». ${ }^{25}$ Selon le gouverneur, qui est à la fois soupçonneux et bon observateur, les Canadiens ont de la difficulté à ne pas trahir leurs sentiments francophiles. ${ }^{26}$ Le procureur général rapporta qu'un écrit signé par le ministre de France aux États-Unis, le citoyen Adet, avait circulé dans le district de Montréal. Adressé aux Canadiens, ce message les assurait que la France, devenue maîtresse de l'Europe continentale, avait maintenant décidé de mettre l'Angleterre à la raison. Elle commencerait d'abord par lui ravir ses colonies. En premier lieu et tout naturellement, les dirigeants de la République française avaient songé aux Canadiens qui gémissaient sous le joug anglais. Ils viendraient bientôt les délivrer de leur esclavage. Le document contenait un exposé éloquent sur les multiples avantages du système républicain que l'on déclarait bien supérieur aux institutions britanniques. Lors de la Révolution américaine, les Canadiens avaient reçu, sous les auspices du Congrès continental, un cours de science politique du même genre. Finalement, cette lettre incendiaire se terminait par la promesse suivante: « On n'entendra bientôt que le cri de Vive la République! depuis le Canada jusqu'à Paris. » ${ }^{27}$

23 Rapport de Sewell, RAC (1891), note D, 75.

24 Prescott à Portland, 24 octobre 1796, ibid., note D, 57-58.

25 Id. à $i d$., 24 octobre 1796 , ibid., note D, 58.

26 Id. à $i d$., 28 octobre 1796, AC, Q 78: 14.

27 Rapport du procureur général au Conseil exécutif, 30 octobre 1796, RAC (1891), note D, 58-59. Il semble bien que les autorités n'ont jamais réussi à saisir un exemplaire de ce manifeste. Sewell s'appuie sur les témoignages qu'il a recueillis pour en exposer la teneur générale. 
Un émissaire qui semble avoir été l'un de ceux qui distribuèrent cette circulaire dans la région de Montréal et qui firent campagne en faveur de la République française annonce, après avoir quitté le Canada, qu'il reviendra au printemps pour faire «danser la carmagnole aux Anglais $\gg .{ }^{28}$

Ce fut à la même époque qu'un correspondant, qui se dit paroissien de Laprairie, envoya un appel au consul de France à New York. ${ }^{29}$ Ce document n'est pas signé quoiqu'il contienne une liste de noms. De plus, il ne porte aucune date. Cependant, vu que son auteur s'adresse à la Convention nationale, qui a gouverné la France du mois de septembre 1792 au mois d'octobre 1795, on peut supposer qu'il a été rédigé avant que ne fût connu au Canada l'établissement du Directoire. Le pétitionnaire énumère quinze personnes qu'il déclare prêtes à donner leur appui aux soldats français qui viendront libérer le pays. Il ajoute les noms de treize pilotes canadiens dont les services pourraient être retenus par la flotte française qui remonterait le fleuve Saint-Laurent.

Des renseignements précis et utiles complètent cet appel. L'auteur donne un tableau des forces anglaises dans la colonie, une liste des magasins militaires et un dénombrement des guerriers sauvages. Selon lui, ceux-ci «sont amis des Canadiens et ne prendront point part pour les Anglais contre la République. On ne pense même pas que ces premiers puissent en corrompre un seul, tellement ils les abhorrent. » Enfin celui qui a pris l'initiative de cette démarche tente de dresser un « Etat des forces de terre et de mer qu'on pense devoir être suffisantes pour la con-

2810 novembre 1796, AC, Q 78: 156-157. Lettre interceptée que le gouverneur Prescott a transmise au gouvernement britannique.

29 - Lettre des Canadiens au consul de la République française à New York 》, AC, Affaires étrangères, Mémoires et documents, Angleterre, 2: 29-36. D'après le classement des Archives publiques du Canada, on suppose que cet appel a été rédigé en 1797 . Je crois qu'il a été écrit après le mois d'août 1795, car l'auteur fait allusion au traité de paix que le général Wayne de l'armée américaine a signé avec les sauvages (traité du fort Greenville, le 3 août 1795), et avant les émeutes de 1796, dont il n'est pas fait mention. 
quête du Canada ». Parmi le matériel réclamé, figurent $\star 20,000$ cocardes nationales $\gg^{30}$

Cet appel des Canadiens à la France révolutionnaire et leur agitation au printemps de 1794 et à l'automne de 1796 peuvent être considérés comme une réponse au message que Genêt avait intitulé «Les Français libres à leurs frères les Canadiens ». ${ }^{\mathbf{3 1}} \mathrm{La}$ lecture de la pétition du paroissien de Laprairie, jointe aux citations données plus haut et aux faits rapportés dans cet article, nous aide à comprendre les sentiments, les espoirs, les frustrations et les calculs qui se bousculaient dans l'esprit de nos ancêtres au moment où la France, qu'ils considéraient toujours comme la mère-patrie et qu'ils ne pouvaient pas ne pas aimer et admirer, triomphait en Europe.

Université de Montréal.

MICHEL BRUNET

\section{LETTRE DES CANADIENS AU CONSUL DE LA REPUBLIQUE FRANÇAISE A NEW YORK}

\section{Citoyen,}

Recevez les vœux de la plus grande partie des Canadiens. Ils aiment tous la France, détestent l'Anglais et désirent ardemment de se voir réunis à la mère-patrie dont ils ont été séparés depuis trop longtemps. Ils voient avec peine que la Convention paraît les oublier. Depuis qu'ils gémissent sous le joug anglais, ces tyrans de jour en jour veulent rendre les fers plus pesants. Les Canadiens voudraient les briser, et pour les mettre à portée de le faire il ne faudrait qu'un coup d'œil favorable et un léger secours de la République, puisqu'il est vrai qu'il y a en Canada plus de 300 opprimés contre un oppresseur. Ces derniers font ce qu'ils peuvent pour dimi-

30 On peut toutefois se demander si tous ces renseignements et toutes ces demandes accompagnaient réellement la lettre proprement dite au consul de New York ou s'ils venaient d'une autre source. D'autre part, il me semble évident qu'une partie au moins de la documentation est due à celui qui a rédigé la lettre au consul.

31 J'en ai publié le texte dans l'article cité à la note (1). 
nuer nos forces qu'ils redoutent au point de former une compagnie de milice d'abord, de la diviser ensuite en deux, puis en quatre pour la rendre plus faible. Mais leurs efforts sont nuls puisque de tous les droits qu'ils nous imposent nous n'en voulons payer aucun et qu'ils n'osent employer la force pour nous y contraindre. Les habitants disent d'une commune voix que leurs pères ont fait serment de fidélité à l'Anglais mais qu'eux ne l'ont pas fait, qu'ils défendront cependant l'Anglais contre tous ses ennemis, excepté contre les Français parce qu'ils ne porteront jamais leurs armes contre leurs pères, leurs frères ou leurs parents. Le plus cher de leurs désirs à présent est de voir l'attention de la France se fixer sur eux. Dès qu'ils en auront la preuve par la présence de la moindre force française, cette force accroîtra de suite de tous les habitants du Canada. Et leurs efforts se porteront contre une poignée impuissante d'Anglais qui ne conservent ce fertile pays que parce que la France a oublié jusqu'à ce moment de s'en ressaisir.

Les citoyens ci-après nommés entraînent avec eux le suffrage de tous nos habitants. Bons patriotes et bons guerriers, ils se réuniront avec les Français qui viendront les arracher au joug anglais. Au premier signal, ils seront suivis de tous ceux qu'il est impossible de nommer ici mais qui ont tous le même courage et le même amour pour la France et pour les Français.

J'ajouterai que tous m'ont chargé d'offrir aux Français leurs cœurs et leurs bras, et que s'ils eussent pu le faire sans risques je serais muni de la signature de tous.

[Suit une liste de noms avec quelques commentaires de peu d'importance.]

(AC, Affaires étrangères, Mémoires et documents, Angleterre, 2: 29-31). 